\title{
Band-Tunable and Multiplexed Integrated Circuits for Simultaneous Recording and Stimulation With Microelectrode Arrays
}

\author{
Roy H. Olsson, III, Member, IEEE, Derek L. Buhl, Anton M. Sirota, Gyorgy Buzsaki, and \\ Kensall D. Wise, Fellow, IEEE
}

\begin{abstract}
Two thin-film microelectrode arrays with integrated circuitry have been developed for extracellular neural recording in behaving animals. An eight-site probe for simultaneous neural recording and stimulation has been designed that includes on-chip amplifiers that can be individually bypassed, allowing direct access to the iridium sites for electrical stimulation. The on-probe amplifiers have a gain of $38.9 \mathrm{~dB}$, an upper-cutoff frequency of $9.9 \mathrm{kHz}$, and an input-referred noise of $9.2 \mu \mathrm{V}_{\mathrm{rms}}$ integrated from $100 \mathrm{~Hz}$ to $10 \mathrm{kHz}$. The low-frequency cutoff of the amplifier is tunable to allow the recording of field potentials and minimize stimulus artifact. The amplifier consumes $68 \mu \mathrm{W}$ from $\pm 1.5 \mathrm{~V}$ supplies and occupies $0.177 \mathrm{~mm}^{2}$ in $3 \mu \mathrm{m}$ features. In vivo recordings have shown that the preamplifiers can record single-unit activity $1 \mathrm{~ms}$ after the onset of stimulation on sites as close as $20 \mu \mathrm{m}$ to the stimulating electrode. A second neural recording array has been developed which multiplexes 32 neural signals onto four output data leads. Providing gain on this array eliminates the need for bulky headmounted circuitry and reduces motion artifacts. The time-division multiplexing circuitry has crosstalk between consecutive channels of less than $6 \%$ at a sample rate of $20 \mathrm{kHz}$ per channel. Amplified, time-division-multiplexed multichannel neural recording allows the large-scale recording of neuronal activity in freely behaving small animals with minimum number of interconnect leads.
\end{abstract}

Index Terms-DC baseline stabilization, neural-recording amplifier, neural recording array, time-division multiplexer.

\section{INTRODUCTION}

$\mathbf{T}$ HE past two decades have shown significant advances in the development of micromachined neural recording arrays. These arrays are now fabricated on a variety of substrates [1]-[6] and are widely used by neurophysiologists to study the nervous system. While passive probes (without integrated circuitry) have been used to record from up to 96 sites simultaneously [7], the large number of interconnects prohibits scaling to higher site counts because of the tethering

Manuscript received June 21, 2004; revised November 18, 2004. This work was supported by National Institutes Health under Contract Number NIH-NINDS-N01-NS-02329. This work made use of the Engineering Research Center for Wireless Integrated Microsystems shared facilities supported by the NSF under Award Number EEC-0096866. Asterisk indicates corresponding author.

*R. H. Olsson, III is with the MEMS Device Technologies Group, Sandia National Laboratories, P.O. Box 5800-1080, Albuquerque, NM 87185-1080 USA (e-mail: rholsso@sandia.gov).

D. L. Buhl, A. Sirota, and G. Buzsaki are with the Center for Molecular and Behavioral Neuroscience, Rutgers University, Newark, NJ 07102 USA (e-mail: dlbuhl@andromeda.rutgers.edu).

K. D. Wise is with the Center for Wireless Integrated MicroSystems, University of Michigan, Ann Arbor, MI 48109 USA (e-mail: wise@ umich.edu).

Digital Object Identifier 10.1109/TBME.2005.847540 forces these interconnects produce on the probe in vivo and the packaging overhead associated with assembling these arrays. In order to provide researchers with access to hundreds or thousands of neurons simultaneously, site selection, time-division multiplexing, and signal processing circuitry must be integrated with the recording arrays to reduce the number of leads. These neural data-compression techniques all require a robust integrated preamplifier capable of amplifying sub-50 $\mu \mathrm{V}$ $(100 \mathrm{~Hz}-10 \mathrm{kHz})$ neural action potentials while rejecting the dc baseline potential of the electrode, which can be as large as $300 \mathrm{mV}$, can drift tens of millivolts, and is highly variable from site to site [1]. When recording from behaving animals, integrated preamplifiers eliminate the need for bulky head-mounted circuitry and have been shown to reduce both stimulation [8] and motion artifacts [9]. Time-division multiplexing can be used to reduce the total number of leads by sampling multiple neural channels onto one data line. It is important to include preamplifiers before the multiplexing to reduce noise caused by clock artifacts and aliasing [1]. The integrated circuitry must be low power such that there is no heating of the surrounding tissue and should be small to reduce tissue trauma and to maintain a low profile above the cortical surface. In addition, these integrated circuits should not contribute large offset voltages to the system, should not degrade the overall signal-to-noise ratio of the neural signals, and should have stable, predictable characteristics.

Recently, progress has been made in the development of both active (with integrated circuitry) neural recording arrays [1], [10] and neural interface circuits [11]-[13]. However, published data with active probes has been limited to electrode arrays with integrated unity-gain buffers [7], [9] because a fully-integrated preamplifier which is immune to both the dc baseline potential of the electrode site and optical currents was unavailable. The lack of a suitable preamplifier has also prevented experiments using time-division-multiplexed neural arrays because of the clock artifacts and aliased noise that corrupt the neural signals when the signals cannot be amplified and filtered before sampling [1].

This paper describes two active neural arrays for recording from behaving animals. The first probe is an eight-site array for simultaneous stimulation and recording in the brain of rats and mice. The probe features eight fully-integrated capacitivelycoupled amplifiers that can be individually bypassed, providing direct access to the iridium sites for neural stimulation. The amplifiers have a tunable low-frequency cutoff for recording ac- 
tion potentials alone $(100 \mathrm{~Hz}-10 \mathrm{kHz})$ or action and field potentials $(10 \mathrm{~Hz}-10 \mathrm{kHz})$ and are insensitive to both the dc polarization of the electrode and optically-induced currents. Tuning the low-frequency cutoff of the amplifier eliminates undesirable low-frequency noise and provides the fastest amplifier settling time for a given application. This amplifier was also included on a 32-channel neural array with integrated time-division multiplexing circuitry. This probe reduces the total number of data leads from 32 to 4 , eliminating many of the tethering forces caused by large numbers of interconnects.

\section{INTEGRATED PREAMPLIFIER DESIGN}

The major challenge in developing fully integrated neuralrecording amplifiers is filtering the low-frequency drift (dc potential) of the electrode while still amplifying neural action potentials and extracellular electroencephalographic fields. This dc baseline stabilization requires pole frequencies at $100 \mathrm{~Hz}$ (action potentials) and $10 \mathrm{~Hz}$ (field potentials), which are not easily realized using integrated components due to the high-value capacitors and resistors needed to achieve these large time constants. The first fully integrated CMOS neural-recording amplifier [14] used diode-capacitor feedback to filter the dc polarization of the electrode. This amplifier was operated in open-loop, which made the gain and output offset voltage difficult to control. Another approach commonly used to filter the dc polarization of the electrode involves loading the recording site with a high resistance so that this resistor and the site capacitance form a high-pass filter. Reverse-biased diodes [1], reset gates [15], and subthreshold-biased transistors [16] have all been implemented to realize this very high resistance. The reverse-biased diodes suffer from optical sensitivity problems that saturate the outputs of the preamplifiers. Using reset gates to realize this high resistance is problematic because of the relatively low reset frequencies required (on the order of $\mathrm{Hz}$ ) and the noise injected by the reset signal. Subthreshold-biased-MOS transistors have recently been explored to realize this high resistance, but these amplifiers have not yet been used in any in vivo experiments [16].

\section{A. Capacitively Coupled Neural-Recording Amplifier}

The capacitively-coupled neural-recording amplifier is shown in Fig. 1. The amplifier uses subthreshold-biased nMOS transistors having an incremental resistance of greater than 10 $\mathrm{G} \Omega$ along with capacitors $\mathrm{C}_{1}$ and $\mathrm{C}_{2}$ to filter the dc baseline potential of the electrode. The gain of the amplifier, $A$, is given by

$$
A=\frac{-R_{\mathrm{NMOS}} C_{1}}{\frac{1}{j \omega}+R_{\mathrm{NMOS}} C_{2}} .
$$

This yields an in-band gain of

$$
\left(\frac{-C_{1}}{C_{2}}\right)=-100
$$

a dc gain of zero, and a lower cutoff frequency of

$$
f_{\mathrm{lc}}=\frac{1}{2 \pi R_{\mathrm{NMOS}} C_{2}}
$$

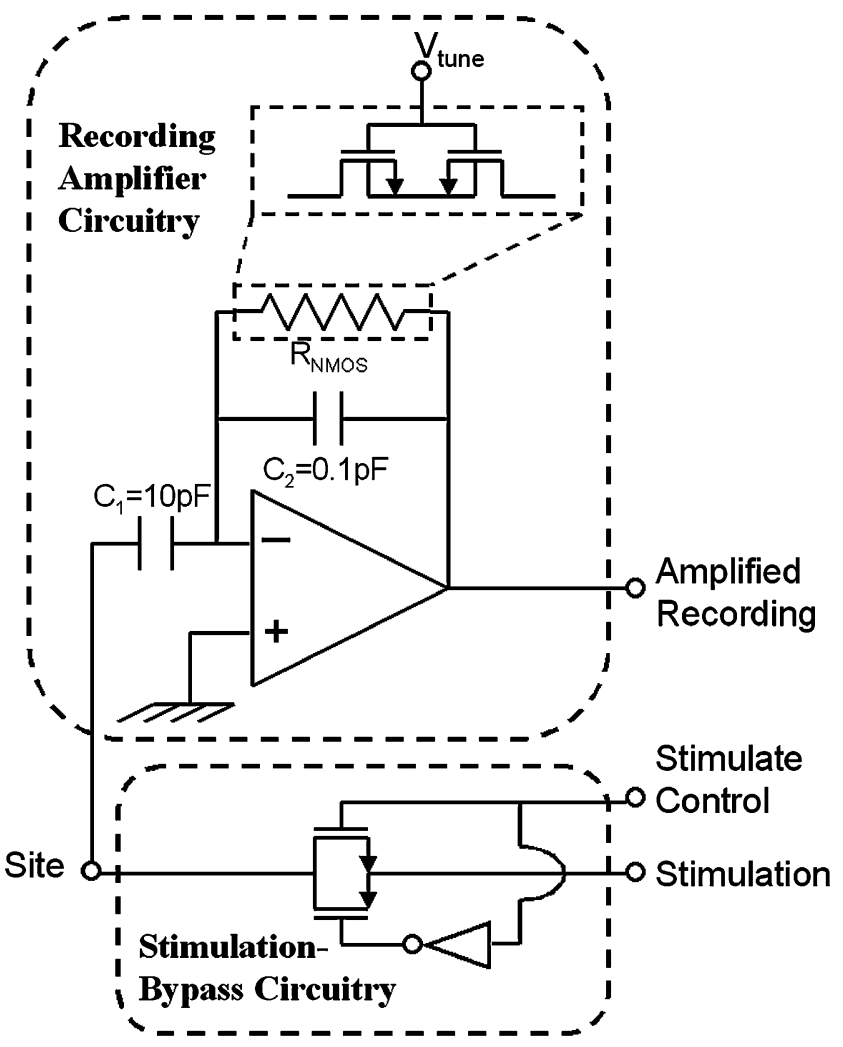

Fig. 1. Capacitively-coupled neural-recording amplifier (top). Circuitry for bypassing the amplifier (bottom) is included on some designs to allow simultaneous stimulation and recording.

for the amplifier shown in Fig. 1. While the site impedance could potentially lower the gain because the double layer capacitance is in series with $\mathrm{C}_{1}$, typical site impedances are two orders of magnitude lower than that of $\mathrm{C}_{1}$. A transistor-level schematic of the amplifier is shown in Fig. 2. This is a miller compensated two-stage amplifier with a source follower output stage. The upper cutoff frequency of the amplifier is given by

$$
f_{h c}=\frac{g_{m} 1}{2 \pi A C_{m}}
$$

where $g_{m 1}$ is the transconductance of M1, $C_{m}$ is the miller capacitance and $A$ is the closed loop gain of the amplifier. The gain of the amplifier shown in Fig. 1 is predictable and reproducible because it is based on a capacitance ratio. Using capacitive rather than resistive feedback is advantageous because high-impedance capacitors can be realized in a small die space and these capacitors draw no dc current. The dc feedback path through $R_{\text {NMOS }}$ ensures a low amplifier output offset voltage by forcing the output offset voltage to be equal to the input offset voltage.

\section{B. Tunable Resistance Subthreshold-Biased MOSFETS}

When performing neurological experiments it is important to ensure the ability to record local field potentials simultaneously with neuron firing. In certain prosthetic applications, however, it may only be necessary to record single-unit activity [17]-[19]. It is therefore desirable to have an integrated preamplifier with a tunable low-frequency cutoff. Precisely tuning the low-frequency cutoff of the preamplifier to the lowest frequency of 


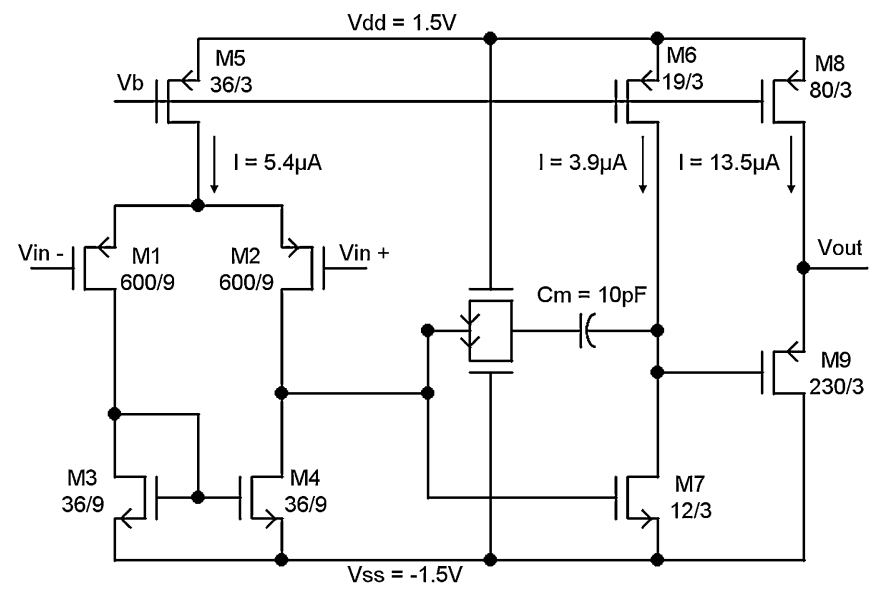

Fig. 2. Transistor-level schematic of the preamplifier.

interest removes undesirable out-of-band noise and yields the fastest settling time for a given application.

The first capacitively-coupled neural-recording amplifier was reported in 2002 [20], followed by a similar design optimized for very-low noise performance [21]. These designs had low-frequency cutoffs of $66 \mathrm{mHz}$ [20], [22], $25 \mathrm{mHz}$ [11] and $0.1 \mathrm{mHz}$ [21] that were highly variable with transistor threshold voltage. While these designs are suitable for wide-band (10 $\mathrm{Hz}-10 \mathrm{kHz}$ ) neural recording they cannot be used in conjunction with implantable neural spike detection circuitry without the addition of an integrated, well controlled high pass filter because low-frequency noise and field potentials will corrupt previously reported spike detection algorithms [23]. Furthermore, due to the large time constants and long settling times associated with sub-60 mHz low-frequency cutoffs, these amplifiers cannot be used to perform simultaneous neural stimulation and recording.

The subthreshold-biased MOSFET's used to implement $R_{\mathrm{NMOS}}$ in Fig. 1 are adjustable via the tuning voltage, $V_{\text {tune }}$. The conductance of a MOSFET operating in the subthreshold region is given by [24]

$$
\frac{d I_{\mathrm{ds}}}{d V_{\mathrm{ds}}}=G_{\mathrm{out}}=\frac{I_{0}}{V_{t}} \exp \left(\frac{V_{\mathrm{gs}}-V_{\mathrm{th}}-V_{\mathrm{off}}}{n V_{t}}\right) \exp \left(\frac{-V_{\mathrm{ds}}}{V_{t}}\right)
$$

where $V_{t}$ is the thermal voltage, $V_{\mathrm{th}}$ is the threshold voltage, $V_{\text {off }}$ is the offset voltage which determines the channel current at $V_{\mathrm{gs}}=0, n$ is the subthreshold swing parameter, and $I_{0}$ is defined in [24]. The exponential dependence of the conductance on threshold voltage and other process parameters that makes the low-frequency cutoff highly variable can be trimmed in this design by adjusting $V_{\text {tune }}$.

\section{Experimental Results}

The measured low-frequency cutoff of the amplifier versus the tuning voltage, $V_{\text {tune }}$, is shown in Fig. 3 . The measured data shows that it is possible to tune the low-frequency cutoff of the amplifier over the desired range $\left(10 \mathrm{~Hz}<\mathrm{f}_{\mathrm{lc}}<500\right.$ $\mathrm{Hz}$ ) for applications where field potentials must be recorded and applications where low-frequency data should be filtered out. While the low-frequency cutoff versus tuning-voltage curve

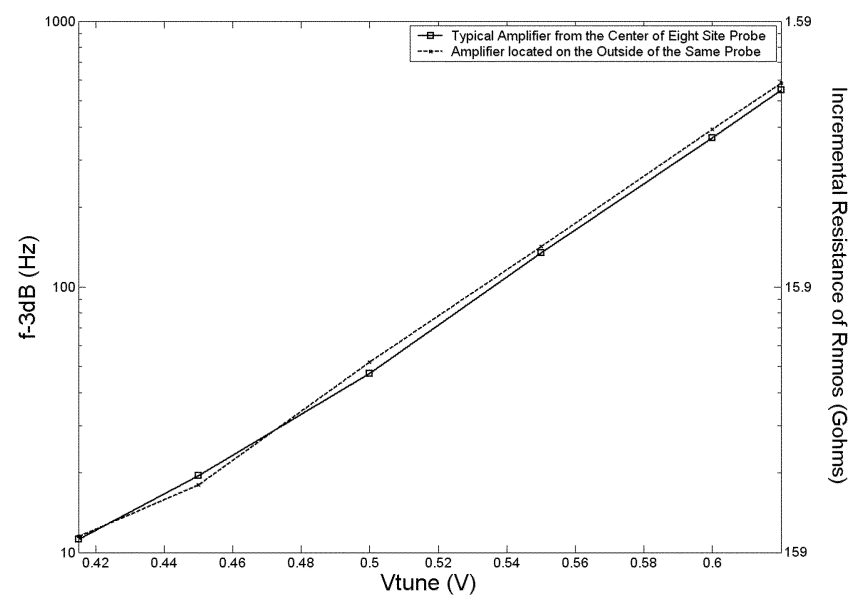

Fig. 3. Amplifier low-frequency cutoff and corresponding $R_{\text {NMOS }}$ incremental resistance versus tuning voltage. The solid curve is the response of a typical preamplifier from the center of this probe. The dashed curved is the measured response from the amplifier furthest $(1.7 \mathrm{~mm})$ from the one represented by the solid line, representing the maximum variation across an eight-site probe. Because the tuning curves for all the amplifiers on a given probe are nearly identical, only one tuning voltage lead is required per probe.
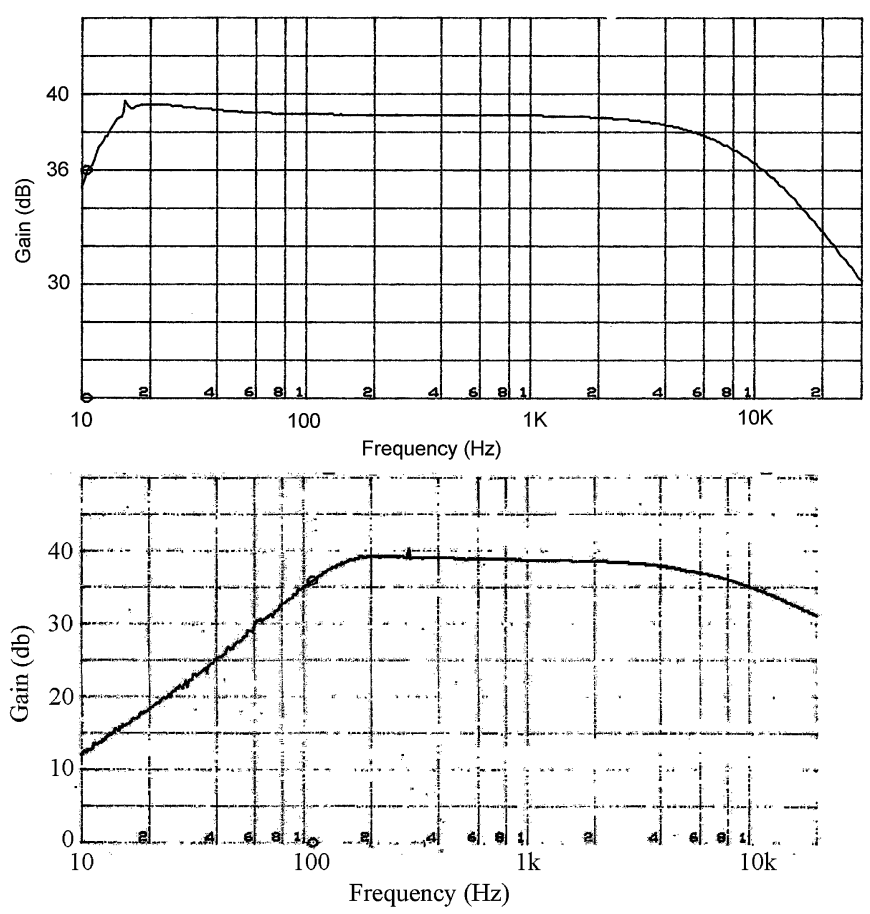

Fig. 4. (Top) Amplifier tuned for a low frequency cutoff of $11 \mathrm{~Hz}$ for recording action and field potentials. (Bottom) Amplifier tuned for a low frequency cutoff of $110 \mathrm{~Hz}$ for recording action potentials while filtering low frequency noise and field potentials.

varies across the wafer and across process runs in accordance with (5), the curves are nearly identical for amplifiers integrated on the same probe (see Fig. 3) allowing for a common bias to be used. Fig. 4 (top) shows the frequency response of the amplifier with $V_{\text {tune }}=0.415 \mathrm{~V}$ to yield a low-frequency cutoff of $11 \mathrm{~Hz}$ for recording field potentials and single units, while Fig. 4 (bottom) shows the amplifier with $V_{\text {tune }}=0.55 \mathrm{~V}$, setting the low-frequency cutoff to $110 \mathrm{~Hz}$ for recording only action potentials. The amplifier has a measured in-band gain of $38.9 \mathrm{~dB}$ and 


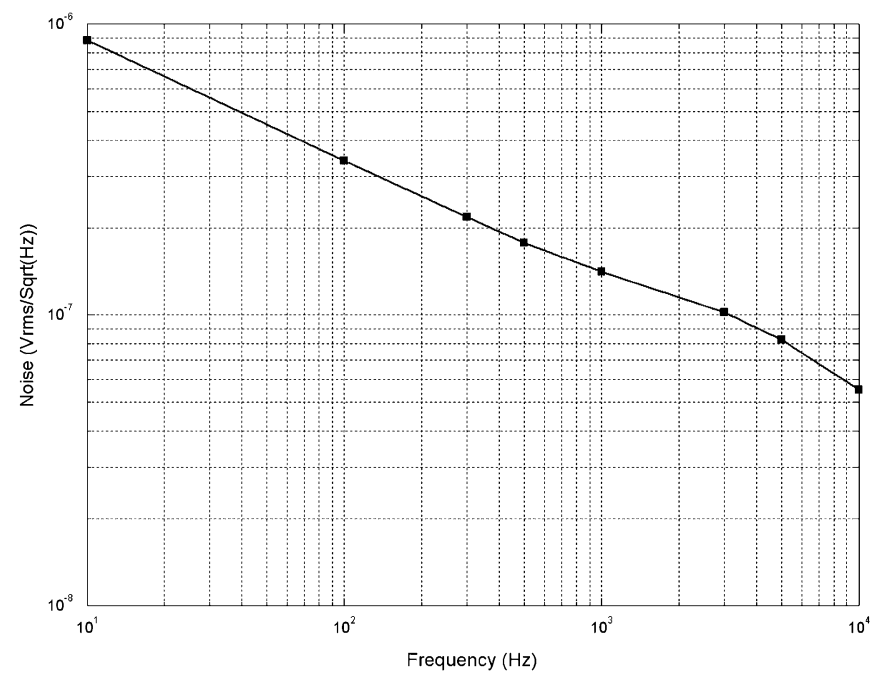

Fig. 5. Input-referred noise spectrum of the capacitively-coupled preamplifier. The total input referred noise integrated from $100 \mathrm{~Hz}$ to $10 \mathrm{kHz}$ is $9.2 \mu \mathrm{V}_{\mathrm{rms}}$. Integrating from $10 \mathrm{~Hz}$ to $10 \mathrm{kHz}$ yields an input referred noise of $10.7 \mu \mathrm{V}_{\mathrm{rms}}$.

an upper cutoff frequency of $9.9 \mathrm{kHz}$. The input-referred noise of the amplifier is shown in Fig. 5. The total input-referred noise integrated from $100 \mathrm{~Hz}$ to $10 \mathrm{kHz}$ is $9.2 \mu \mathrm{V}_{\mathrm{rms}}$, which is lower than that of a $165 \mu \mathrm{m}^{2}$ iridium recording site in saline [1]. When recording in wide-band mode, $10 \mathrm{~Hz}$ to $10 \mathrm{kHz}$, the total integrated noise is $10.7 \mu \mathrm{V}_{\mathrm{rms}}$. The noise performance is limited primarily by transistors $M 1-M 4$ in Fig. 2 [22]. The noise contributed by the feedback transistors in Fig. 1 is negligible as the input referred current sources of these transistors are shorted to $\mathrm{AC}$ ground and the input referred voltage noise generators of these transistors are attenuated by greater than $60 \mathrm{~dB}$ in band when compared to the voltage noise generators of M1 and M2 in Fig. 2. Table I summarizes the performance of the integrated circuitry.

\section{Simultaneous Neural Stimulation AND Recording}

\section{A. Design and Operation}

An eight-site neural recording array designed to permit simultaneous stimulation and recording is shown in Fig. 6 (top). These arrays were fabricated using the process described by Bai [1] modified to include a 6 - $\mu \mathrm{m}$-thick gold shield electroplated over the circuitry to insulate it from the biological environment. The $165 \mu \mathrm{m}^{2}$ iridium recording sites are located at the tip of the shank, which is $5 \mathrm{~mm}$ in length, approximately $15 \mu \mathrm{m}$ thick, and tapers from a width of $52 \mu \mathrm{m}$ at the base to a sharp tip. The back-end of the probe, where the integrated electronics and bonding pads are located, is $3.26 \mathrm{~mm}$ wide and $2.66 \mathrm{~mm}$ long. When the Stimulate Control signal in Fig. 1 is low, the switch connecting the Site to the Stimulation pad is open and active neural recording is performed on that channel. When the Stimulate Control signal is high, the switch is closed and there is an ohmic connection between the Site and the Stimulate pad where a stimulation current or voltage can be coupled into the tissue. Neural recording can still be performed while the switch is closed (Stimulate Control is high); however, many of the advantages of recording with integrated amplifiers such as reduced crosstalk, packaging requirements, and spurious
TABLE I

PERFormance of THE INTEGRATED AMPLIFIER AND MultiPleXer CirCuitry

\begin{tabular}{c|c}
\hline Gain & $38.9 \mathrm{~dB}$ \\
\hline Bandwidth & Tunable $<\mathrm{f}_{0}<9.9 \mathrm{kHz}$ \\
\hline DC gain & 0 \\
\hline DC Rejection Range & $> \pm 500 \mathrm{mV}$ \\
\hline Supply Voltage & $\pm 1.5 \mathrm{~V}$ \\
\hline Power Consumption & $68 \mu \mathrm{W}$ \\
\hline Die Area (3 $\mu \mathrm{m}$ features) & $0.177 \mathrm{~mm}^{2}$ \\
\hline $\begin{array}{c}\text { Input-referred Noise Voltage Integrated from } \\
\text { 100 Hz to 10 kHz }\end{array}$ & $9.2 \mu \mathrm{V}_{\mathrm{rms}}$ \\
\hline $\begin{array}{c}\text { Output Impedance (Dominated by } \\
\text { Interconnect) }\end{array}$ & $<1 \mathrm{k} \Omega$ \\
\hline Output Offset Voltage & $-45 \mathrm{mV}$ \\
\hline PSRR at 1 kHz & $-50.5 \mathrm{~dB}$ \\
\hline $\begin{array}{c}\text { Multiplexer Crosstalk Between Consecutive } \\
\text { Channels (SR }=20 \mathrm{kHz} / \text { channel) }\end{array}$ & $<6 \%$ \\
\hline
\end{tabular}

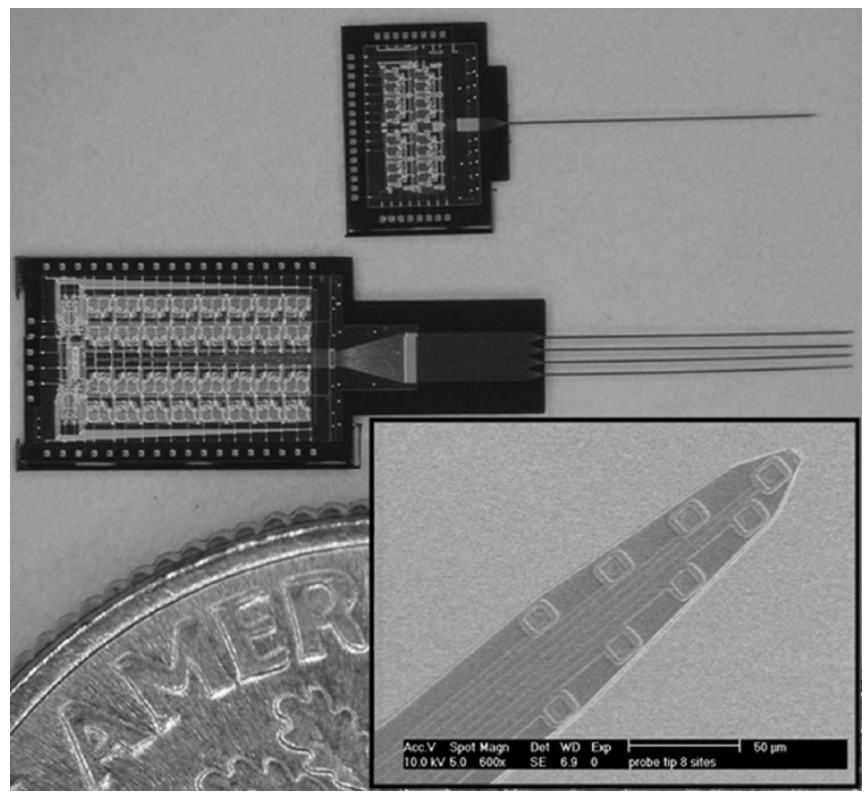

Fig. 6. An eight-site neural array for simultaneous stimulation and recording (top). Thirty-two channel neural array with integrated amplification and time-division multiplexing circuitry (bottom). Inset shows the dimensions and site spacing on all of the shanks.

noise pick-up will be negated. The use of a CMOS switch to access the site limits the stimulation voltages to values between $\mathrm{Vdd}+0.7 \mathrm{~V}$ and $\mathrm{Vss}-0.7 \mathrm{~V}$. Modeling the site as a $300 \mathrm{pF}$ capacitor limits the total amount of charge that can be delivered or extracted from the tissue to $660 \mathrm{pC}$. The CMOS switches, however, are not limiting the total amount of charge that can be transferred to the tissue in this application, as increasing the voltage across the site in excess of $1 \mathrm{~V}$ will violate the water window of the electrode. Ohmic access to each site can be controlled individually, providing 256 separate stimulation combinations.

\section{B. In Vivo Results}

The eight channel neural array was implanted in the neocortex and hippocampus of rats. The close spacing of the recording sites (20-40 $\mu \mathrm{m}$ center-to-center) allowed for simultaneous recording from the same sets of neurons. The co-occurrence of neuronal spikes on several sites made it possible to designate one of the sites as the stimulation channel (recorded data not shown for this site) while the remaining sites were used for recording. Fig. 7(A) illustrates a short segment 
A

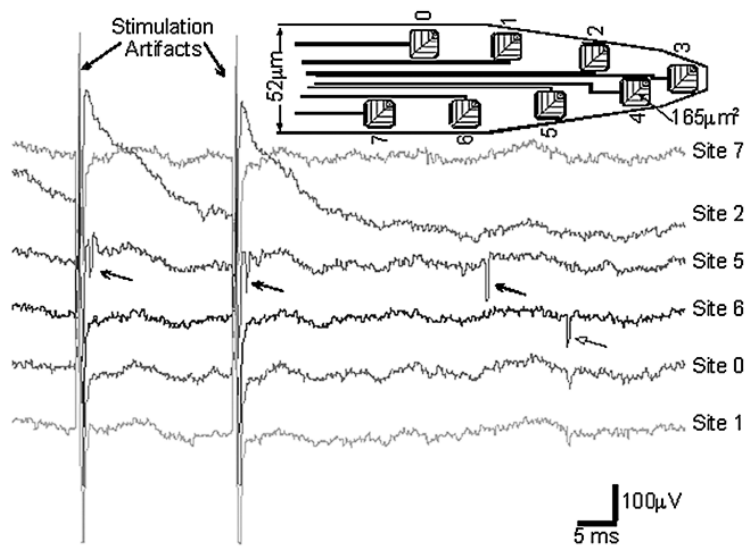

B

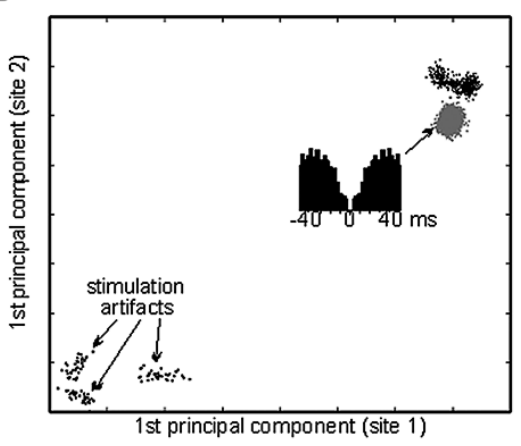

C

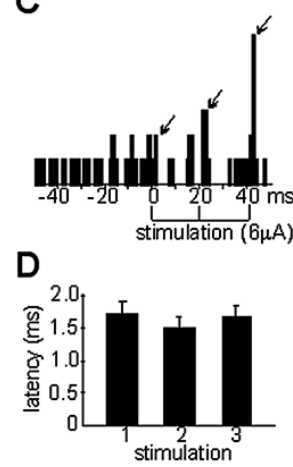

Fig. 7. Simultaneous recording and stimulation with a silicon probe in the CA1 pyramidal layer of the rat. (A) Example of stimulated neuron. Site 4 was the stimulation site and site 3 did not record. Site 2 had a large impedance, as reflected by the increased artifact. Note that triple pulse (first stimulation pulse not shown) local stimulation selectively discharged the neuron on the third trace within $2 \mathrm{~ms}$ (solid arrows, latency shown in (D)). Open arrow, a different neuron recorded by three sites that was not affected by the stimulation. (B) Principal component analysis of the units and stimulation artifacts shown in (A). Note that using PCA, each stimulation event can be isolated as well as the spontaneous activity of the stimulated neuron. (C) Peri-stimulus histogram of the stimulated neuron. Time zero is the onset of the first of three stimulation events. Note that the highest probability of response is to the third stimuli. (D) Mean/STD latency of response to individual stimulation events.

of wide-band $(10 \mathrm{~Hz}-10 \mathrm{kHz})$ recordings. One to three $100 \mu \mathrm{s}$ stimulation pulses reliably discharged one of the recorded units within 2 ms [Fig. 7(D)], whereas it had no effect on another unit recorded further away from the stimulation site. Although the stimulus artifact lasted for 1-2 ms, depending on stimulus strength $(4-8 \mu \mathrm{A})$, it was sufficiently short to allow for the visualization of the evoked unit firing.

The close spacing of the sites also allowed the isolation of putative neurons using principal component analysis [Fig. 7(B)] previously described by Csicsvari [25], [26]. This method allows the identification and isolation of individual neurons from the extracted action potentials based on amplitudes and wave shapes. After unit separation, post-stimulus histograms were constructed for each unit. Fig. 7(C)-(D) illustrates that one of the units could be directly excited by electrical stimulation $(6$ $\mu \mathrm{A})$. The response probability shown in Fig. 7(C) increased with stimulus repetition, reaching the highest probability after the third pulse with a latency of $1.67 \pm 0.18 \mathrm{~ms}$. Other nearby neurons were not affected by the stimulation, indicating that the neuron was directly, rather than synaptically, activated.

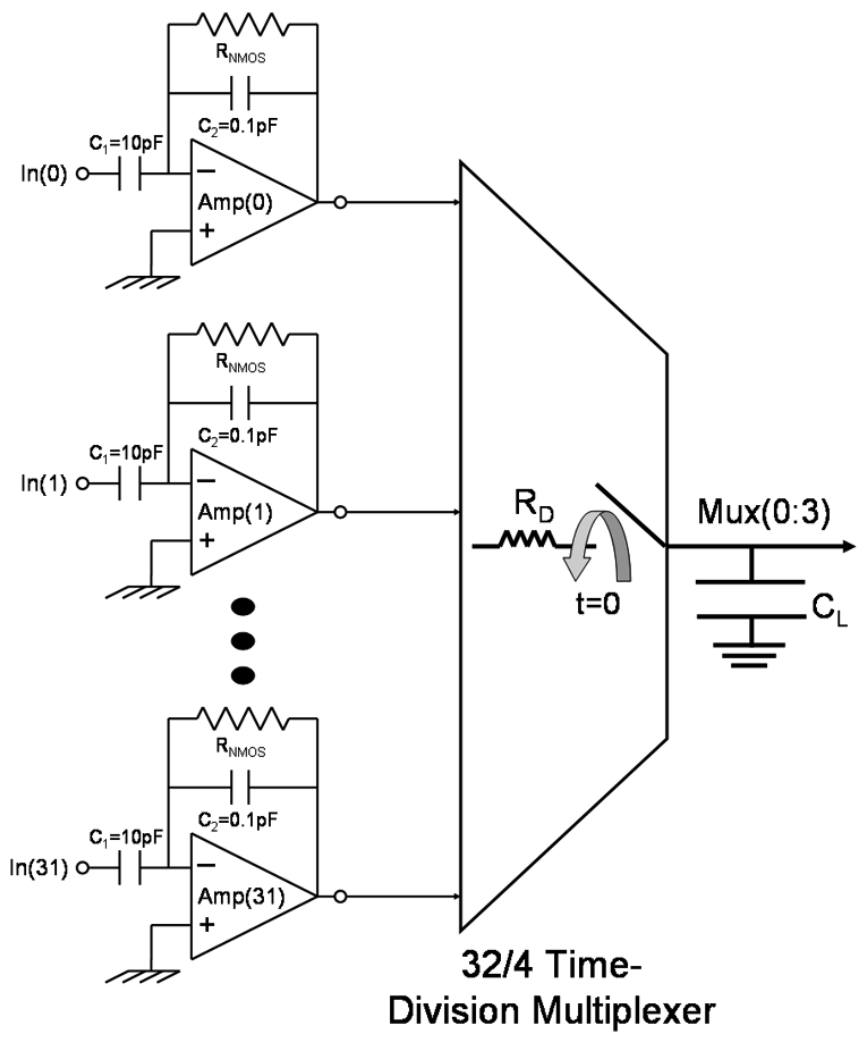

Fig. 8. Block diagram of the 32 channel neural recording probe circuitry, consisting of amplifiers and time-division multiplexers. These circuits have been integrated on the probe in Fig. 6 (bottom).

\section{MultiPleXed NeURAl ReCORDING ARRAY}

With the increasing demand to record from more neurons simultaneously, on-probe data compression techniques are required. Time-division multiplexing reduces the number of data leads by sampling multiple channels onto a single line. This reduces both the overall size of the implant and the packaging requirements. The only in vivo time-division-multiplexed recordings were reported by Bai [1]. In this experiment, source-follower buffers were used to transform the site impedance before four channels of neural data were multiplexed onto a single data line. Bai investigated the noise contributed by multiplexing in vitro and found that the site, on-probe buffers, external electronics and multiplexing clock (reset was held high so no sampling was performed) contributed $9 \mu \mathrm{V}_{\text {rms }}$ of input-referred noise integrated from $300 \mathrm{~Hz}$ to 3 $\mathrm{kHz}$. When the signal was sampled, the total integrated noise increased to $15 \mu \mathrm{V}_{\mathrm{rms}}$, indicating that $12 \mu \mathrm{V}_{\mathrm{rms}}$ of noise was aliased into the signal band since no low-pass filtering was provided before the multiplexing. This result supports the need for preamplification and filtering prior to sampling and limits the noise performance of chopper-modulated neural-recording amplifiers [27].

A 32-site neural-recording array with integrated amplifiers and time-division multiplexing circuitry is shown in Fig. 6 (bottom), with a block diagram of the on-probe circuitry shown in Fig. 8. The on-probe amplifiers provide a gain of $40 \mathrm{~dB}$ and band-limit the signal to $9.9 \mathrm{kHz}$ to prevent aliasing. Each of the 32 neural channels are then sampled at $20 \mathrm{kHz}$ per channel and 
multiplexed onto four data leads. The time-division multiplexer switches channels on both the rising and falling edges of the clock to prevent clock transitions in the middle of the sampling window. The multiplexing requires two additional control leads, clock and reset, for a total of 10 leads. The potential for including on-chip clock and reset generation has been demonstrated [14].

\section{A. Multiplexer Crosstalk}

Shown in Fig. 8 is a circuit schematic representing the output of the time-division multiplexer where the multiplexer switches channels at time $t=0$. There are two processes limiting how quickly the time-division multiplexer can settle from the previous output value, $V_{\text {out }}(n-1)$, to the current output value, $V_{\text {out }}(n)$.

The limited slew rate of the amplifiers shown in Fig. 8 can lead to crosstalk between consecutive channels. The amount of time required to slew the output of the time-division multiplexer to $V_{\text {out }}(n), t_{\text {slew }}$, is given by

$$
t_{\text {slew }}=\frac{C_{L}\left(V_{\text {out }}(n)-V_{\text {out }}(n-1)\right)}{I_{\mathrm{amp}}}
$$

where

$$
\mathrm{V}_{\text {out }}(n)=A^{*} V_{\text {neuron }}+V_{\text {offset }}
$$

$C_{L}$ is the capacitance that loads the output of the time-division multiplexer and $I_{\mathrm{amp}}$ is the maximum output current of the amplifier driving the time-division multiplexer, $14 \mu \mathrm{A}$ for the amplifier shown in Fig. 2. Since the time-division multiplexer samples eight neural channels onto one data line at 20 $\mathrm{kHz}$ per channel and assuming that the output of the multiplexer will be sampled two thirds of the way through the sampling window, yields a maximum allowable $t_{\text {slew }}$ of $4.2 \mu \mathrm{s}$. Given a maximum value of $V_{\text {out }}(n)-V_{\text {out }}(n-1)$ of $100 \mathrm{mV}$ (50 $\mathrm{mV}$ representing neural spike activity at the output of the amplifier and $50 \mathrm{mV}$ representing potential differences in offset voltages between amplifiers on consecutive channels) and a maximum load capacitance of $10 \mathrm{pF}$, the maximum number of channels that can be sampled onto one data lead based on slew-rate limitations is 467 . Unfortunately, amplifier slew rates do not limit the speed and load capacitances of most practical multiplexed neural recording systems. However, there is a clear power consumption limit, represented by $I_{\mathrm{amp}}$ in (6), for neuralrecording amplifiers driving time-division multiplexed systems given the load capacitance, sampling rate, the variability in amplifier offset voltages, and the gain of the preamplifier.

In this sampled neural recording system the time constant formed by $C_{L}$, the load capacitance of time-division multiplexer, and $R_{D}$, the parasitic interconnect resistance, switch resistance, and amplifier output resistance, dominate the settling time of time-division multiplexers. Here, the output voltage $V_{\text {out }}(t)$ is given by

$$
\begin{aligned}
\mathrm{V}_{\text {out }}(t)=\left(A^{*} V_{\text {neuron }}+V_{\text {offset }}\right)(1- & \left.e^{-t / R_{D} C_{L}}\right) \\
& +V(0) e^{-t / R_{D} C_{L}} .
\end{aligned}
$$

It is clear that increasing the sampling frequency or load capacitance not only increases the crosstalk from the previous channel, $V(0)$, but also attenuates the signal from the present channel. Thus the effective crosstalk between consecutive channels can be derived as

$$
\mathrm{CT}=\frac{e^{-K / p f_{s} R_{D} C_{L}}}{1-e^{-K / p f_{s} R_{D} C_{L}}}
$$

where $p$ is the number of channels sampled by the multiplexer, $f_{s}$ is the sample rate per channel and $K$ is the point in the sampling window when $V_{\text {out }}(t)$ is measured, where $0<K<1$. The numerator in (9) represents the voltage at the output due to the previous channel voltage stored across the multiplexer load capacitor prior to switching channels while the denominator is the output voltage due to that of the desired or present channel. While the traditional definition of crosstalk only models the numerator it is important to also include the denominator term because sampling too quickly leads to attenuation of the desired or present channel. This effectively increases the crosstalk because the voltage from the previous channel is essentially amplified (the desired signal is attenuated by the denominator) when referred to the multiplexer input. Holding the crosstalk to less than $5 \%$, given $K=0.66, R_{D}=90 \mathrm{k} \Omega, C_{L}=10 \mathrm{pF}$, and $f_{s}=20$ $\mathrm{kHz}$, limits the number of channels per data lead to 12 .

$$
\text { channels } / \text { lead }=p=\frac{-K}{f_{s} R_{D} C_{L} \ln \left(\frac{\mathrm{CT}}{1+\mathrm{CT}}\right)} \text {. }
$$

Shown in Fig. 9 (top) is the measured crosstalk between consecutive multiplexer channels versus input voltage magnitude at a sampling frequency of $20 \mathrm{kHz} / \mathrm{channel}$, showing the time-constant and slew-rate limited regions. The crosstalk between consecutive channels versus per channel sampling frequency with the input-signal magnitude equal to $1.5 \mathrm{mVpp}$ is shown in Fig. 9 (bottom). Both of these measurements were taken with the multiplexer driving a $0.1 \mathrm{~m}$ cable to an off-chip buffer. The measured data indicates that the on-probe neural recording circuitry can multiplex eight neural channels onto a single data lead at a sampling rate of $20 \mathrm{kHz} / \mathrm{channel}$ with a crosstalk between consecutive channels of less than $6 \%$ provided that the external cable used to interface to the electrode is less than $10 \mathrm{~cm}$ and that the neural action potentials do not exceed $1.5 \mathrm{mVpp}$.

\section{B. In Vivo Recordings}

Fourteen nonmultiplexed simultaneous wide-band neural recordings measured from the outputs of the preamplifiers driving $8.75 \mathrm{~m}$ of cable are shown in Fig. 10 (top). The same fourteen channels recorded 1 minute later with $V_{\text {tune }}$ adjusted to eliminate low-frequency field potentials and noise are shown in Fig. 10 (bottom), verifying that the tunable amplifier can record both wide-band neural data and can eliminate low frequencies when spike detection is to be performed without distorting the action potentials. Three (of the eight) channels of time-division multiplexed neural recordings are shown in Fig. 11. The sampling rate of the time-division multiplexer here is $2.5 \mathrm{kHz} / \mathrm{channel}$. Due to the length of the cable driven 

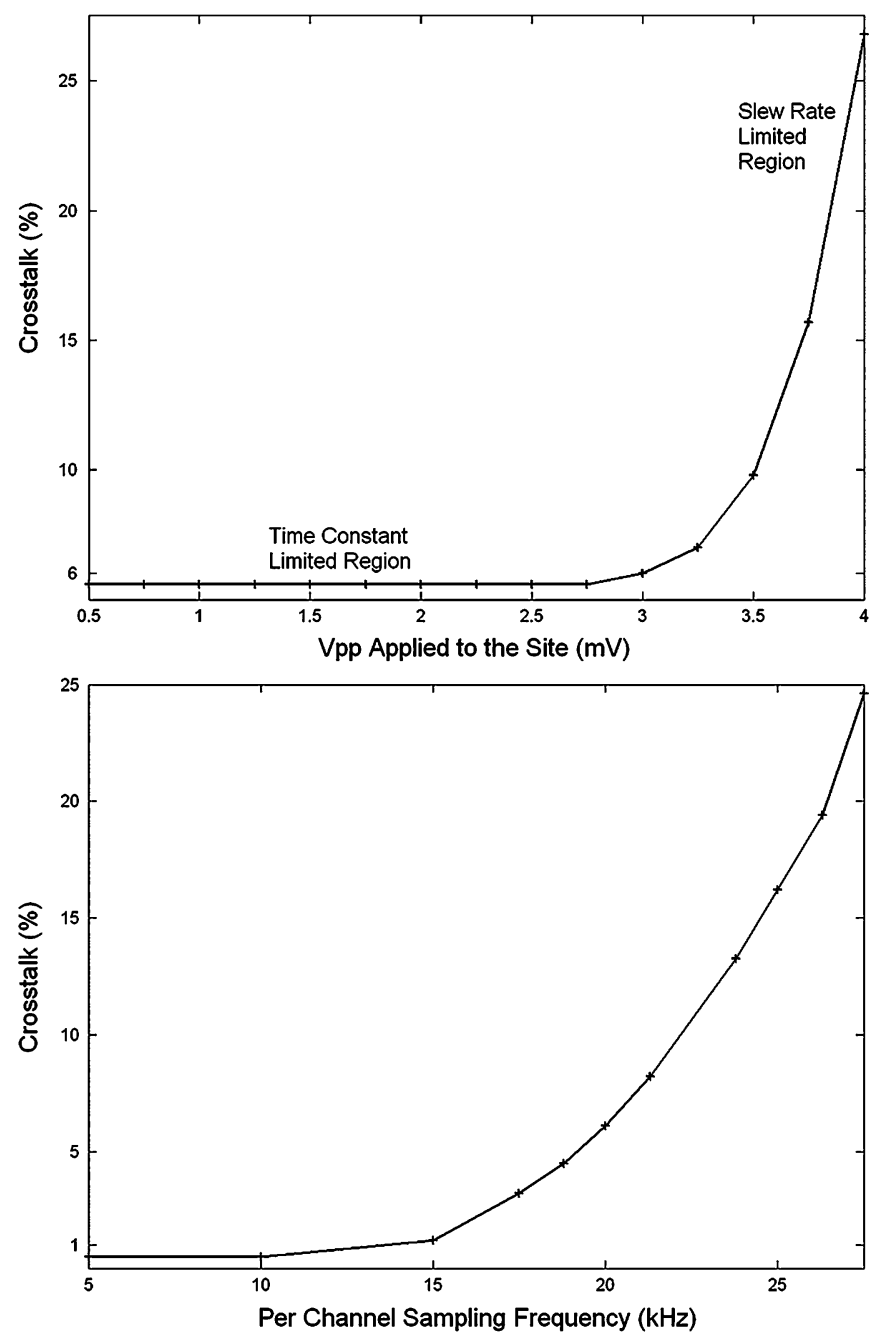

Fig. 9. Multiplexer crosstalk between consecutive channels versus input voltage level at a sampling frequency of $20 \mathrm{kHz} / \mathrm{channel}$ (top). Multiplexer crosstalk between consecutive channels versus per channel sampling frequency with an input voltage of $1.5 \mathrm{mVpp}$ (bottom). The input signal is applied to the site and passed through the amplifier before reaching the time-division multiplexer.

by the time-division multiplexer, $2.4 \mathrm{~m}$, sample rates above $5 \mathrm{kHz} /$ channel resulted in signal attenuation and crosstalk in accordance with (8) and (9). The signals were demultiplexed using Lab View after being sampled and digitized using a 6062E National Instruments data acquisition card.

In the current design, the sampling frequency of the time-division multiplexer is being limited by the load capacitance of the cable and the resistance of the switches (approx. $90 \mathrm{k} \Omega$ ) used to perform the multiplexing. In order to drive the longer cables required for free moving animal experiments, buffers can be mounted on the head of the animal, reducing the cable length and capacitive loading at the output of the time-division multiplexer. In future designs, these buffers will be included on-chip, reducing the output resistance of the time-division multiplexer to below $100 \Omega$. By placing a buffer between the large switch resistance used to perform the multiplexing and the large capacitance introduced by the cable, it is possible to drive up to $10 \mathrm{~m}$ of cable while multiplexing 8 channels onto a single data lead at $20 \mathrm{kHz} / \mathrm{channel}$.

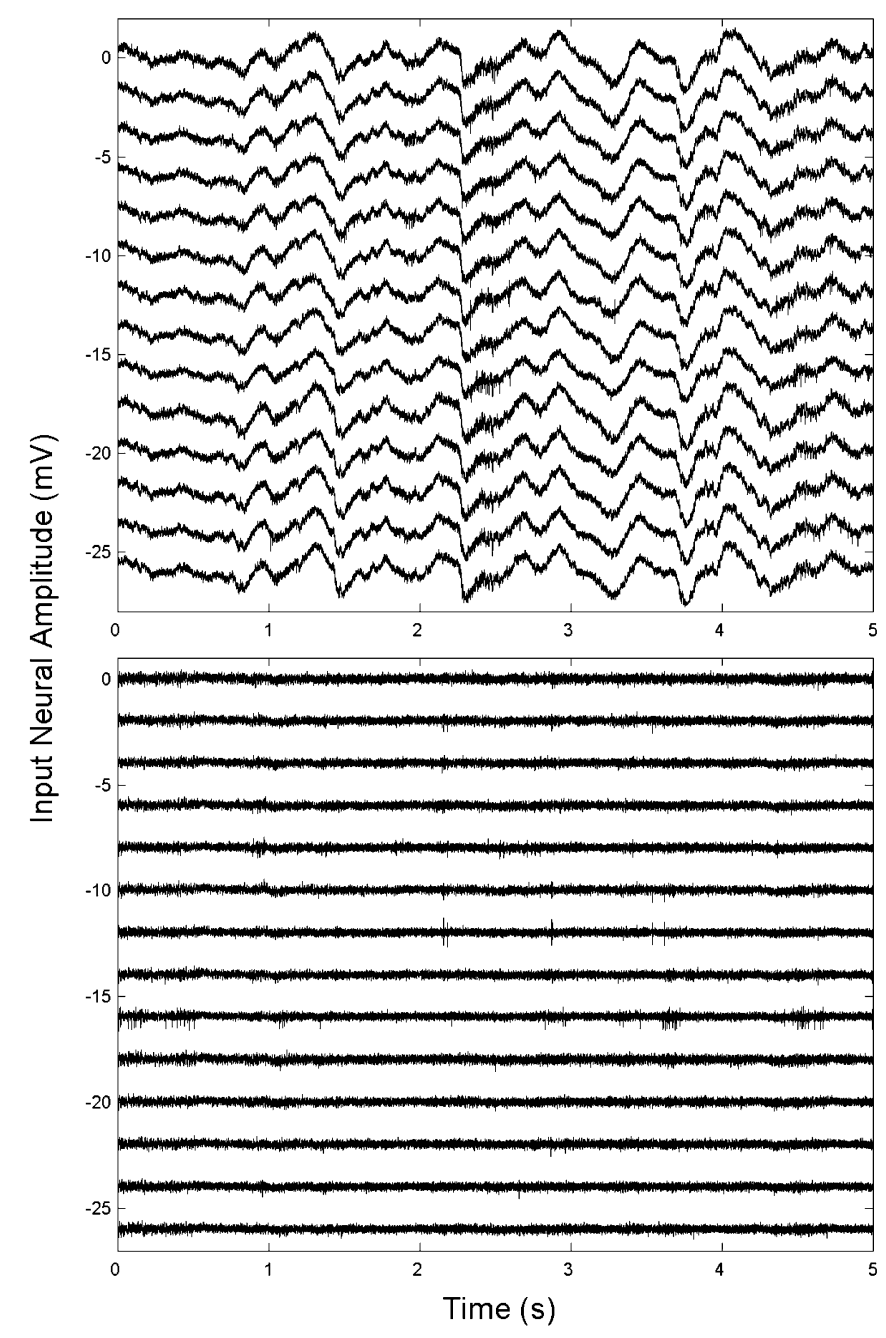

Fig. 10. Fourteen nonmultiplexed channels of wide-band neural data recorded from rat hippocampus using the 32-site array (top). The same fourteen nonmultiplexed channels recorded one minute later with $V_{\text {tune }}$ adjusted to record only action potentials (bottom).

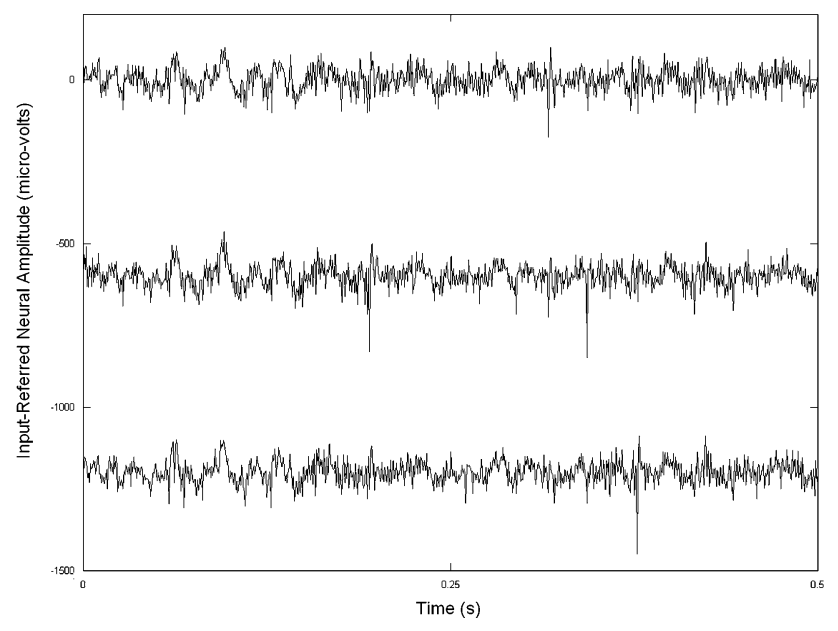

Fig. 11. Time-division multiplexed neural potentials recorded from rat hippocampus. The signals were demultiplexed using Lab View and a data acquisition card.

\section{CONCLUSION}

Two neural recording arrays with integrated circuitry for studying the brains of behaving animals have been developed. 
Both electrodes feature a capacitively-coupled neural-recording amplifier that eliminates the dc baseline potential of the electrode, is not optically sensitive, and has a tunable low-frequency cutoff. The capacitively-coupled neural-recording amplifier has successfully recorded wide-band neural field and action potentials and has been used to eliminate low-frequency field potentials while still recording action potentials, as will be necessary for applications involving implantable spike detection for motor prostheses. An eight-channel neural recording array for simultaneous recording and stimulation has been developed. In vivo recordings have shown that the preamplifiers can record single-unit activity $1 \mathrm{~ms}$ after the onset of stimulation on sites as close as $20 \mu \mathrm{m}$ to the stimulating electrode. A 32-channel neural-recording array with integrated amplifiers and time-division multiplexing circuitry has been developed and implanted. The total crosstalk between consecutive channels at a sampling rate of $20 \mathrm{kHz} / \mathrm{channel}$ is less than $6 \%$ when driving less than $10 \mathrm{~cm}$ of cable. In vivo time-division multiplexed recordings have been obtained at sample rates of up to $5 \mathrm{kHz} /$ channel. Continued work to reduce the capacitance loading the time-division multiplexer circuitry will enable sample rates of greater than $20 \mathrm{kHz} / \mathrm{channel}$, even when driving the long cables necessary for recording from behaving animals.

\section{ACKNOWLEDGMENT}

The authors wish to thank Dr. W. Heetderks of the Neural Prosthesis Program, National Institutes of Neurological Disorders and Stroke, for his encouragement and support of this work under NIH Contract Number NIH-NINDS-N01-NS-02 329. This work made use of the Engineering Research Center for Wireless Integrated Microsystems shared facilities supported by the NSF under Award Number EEC-0 096 866. The assistance of Ning Gulari, Dr. Brian Jamieson, Dr. Andrew DeHennis, Brendan Casey, Robert Gordenker, and Jamie Hetke was also very much appreciated.

\section{REFERENCES}

[1] Q. Bai and K. D. Wise, "Single-unit neural recordings with active microelectrode arrays," IEEE Trans. Biomed. Eng., vol. 48, no. 8, pp. 911-920, Aug. 2001.

[2] P. K. Campbell, K. E. Jones, R. J. Huber, K. W. Horch, and R. A. Normann, "A silicon-based, three-dimensional neural interface: Manufacturing processes for an intracortical electrode array," IEEE Trans. Biomed. Eng., vol. 38, no. 8, pp. 758-768, Aug. 1991.

[3] J. J. Burmeister, K. Moxon, and G. A. Gerhardt, "Ceramic-based multisite microelectrodes for electrochemical recordings," Analytical Chem., vol. 72, no. 1, pp. 187-192, 2000.

[4] P. Norlin, M. Kindlundh, A. Mouroux, K. Yoshida, and U. G. Hofmann, "A 32-site neural recording probe fabricated by DRIE of SOI substrates," J. Micromech. Microeng., vol. 12, no. 4, pp. 414-419, 2002.

[5] K. Cheung, G. Lee, K. Djupsund, Y. Dan, and L. P. Lee, "A new neural probe using SOI wafers with topological interlocking mechanisms," in Proc. IEEE-EMBS Special Topic Conf. Microtechnologies in Medicine and Biology, Lyon, France, 2000, pp. 507-511.

[6] P. J. Rousche, D. S. Pellinen, D. P. Pivin, J. C. Williams Jr., R. J. Vetter, and D. R. Kipke, "Flexible polyimide-based intracortical electrode arrays with bioactive capability," IEEE Trans. Biomed. Eng., vol. 48, no. 3, pp. 361-371, Mar. 2001.
[7] J. Csicsvari, B. Jamieson, K. D. Wise, and G. Buzsaki, "Mechanisms of gamma oscillations in the hippocampus of the behaving rat," Neuron, vol. 37, no. 2, pp. 311-322, 2003.

[8] R. H. Olsson III, D. Buhl, M. N. Gulari, G. Buzsaki, and K. D. Wise, "A silicon microelectrode array for simultaneous recording and stimulation in the brain of behaving animals," in Proc. 25th Annu. Meeting Engineering in Medicine and Biology Society, Cancun, Maxico, 2003, pp. 1968-1971.

[9] J. Csicsvari, D. A. Henze, B. Jamieson, K. D. Harris, A. Sirota, P. Bartho, K. D. Wise, and G. Buzsaki, "Massively parallel recording of unit and local field potentials with silicon based electrodes," J. Neurophys., vol. 90, no. 2, pp. 1341-1323, 2003.

[10] M. D. Hills, D. T. Kewley, J. M. Bower, and G. T. A. Kovacs, "Active SOI-based neural probes," in Proc. Solid State Sensor, Actuator and Microsystems Conf., Hilton Head, SC, 2002, pp. 193-196.

[11] R. R. Harrison and C. Charles, "A low-power low-noise CMOS amplifier for neural recording applications," IEEE J. Solid State Circuits, vol. 38, no. 6, pp. 958-965, Jun. 2003.

[12] P. Mohseni and K. Najafi, "A low-power fully-integrated bandpass operational amplifier for neural recording applications," in Proc. 2nd Joint EMBS/BMES Conf., Houston, TX, 2002, pp. 2111-2112.

[13] I. Obeid, J. C. Morizio, K. A. Moxon, M. A. L. Nicolelis, and P. D. Wolf, "Two multichannel integrated circuits for neural recording and signal processing," IEEE Trans. Biomed. Eng., vol. 50, no. 2, pp. 255-258, Feb. 2003.

[14] J. Ji and K. D. Wise, "An implantable CMOS circuit interface for multiplexed microelectrode recording arrays," IEEE J. Solid State Circuits, vol. 27 , no. 3, pp. 433-443, 1992.

[15] J. L. Lund, "Performance characteristics of active silicon microelectrode arrays," Ph.D. dissertation, Dept. Electrical Engineering and Computer Science, Univ. Michigan, Ann Arbor, 1996.

[16] P. Mohseni and K. Najafi, "A fully integrated neural-recording amplifier with dc input stabilization," IEEE Trans. Biomed. Eng., vol. 51, no. 5, pp. 832-837, May 2004.

[17] D. M. Taylor, S. I. Helms Tillery, and A. B. Schwartz, "Direct cortical control of 3-D neuroprosthetic devices," Science, vol. 296, no. 5574, pp. 1829-1832, 2002.

[18] M. D. Serruya, N. G. Hatsopoulos, L. Paninski, M. R. Fellows, and J. P. Donoghue, "Instant neural control of a movement signal," Nature, vol. 416, no. 6877, pp. 141-142, 2002.

[19] J. M. Carmena, M. A. Lebedev, R. E. Crist, J. E. O’Doherty, D. M. Santucci, D. F. Dimitrov, P. G. Patil, C. S. Henriquez, and M. A. L. Nicolelis, "Learning to control a brain-machine interface for reaching and grasping by primates," PLoS Biol., vol. 1, no. 2, pp. 1-16, 2003.

[20] R. H. Olsson, III, M. N. Gulari, and K. D. Wise, "Silicon neural recording arrays with on-chip signal processing for in vivo data acquisition," Proc. IEEE-EMBS Special Topic Conf. Microtechnologies in Medicine and Biology, pp. 237-240, 2002.

[21] R. R. Harrison, "A low-power, low-noise CMOS amplifier for neural recording applications," in Proc. IEEE Int. Symp. Circuits and Systems, vol. 5, 2002, pp. 197-200.

[22] R. H. Olsson III, M. N. Gulari, and K. D. Wise, "A fully integrated bandpass amplifier for extracellular neural recording," in Proc. 1st Int. IEEE EMBS Conf. Neural Engineering, Capri, 2003, pp. 165-168.

[23] R. R. Harrison, "A low-power integrated circuit for adaptive detection of action potentials in noisy signals," in Proc. 25th Annu. Meeting Engineering in Medicine and Biology Society, Cancun, Mexico, 2003, pp. $3325-3328$.

[24] X. Xi, K. M. Cao, H. Wan, M. Chan, and C. Hu, BSIM4.2.1 MOSFET Model User's Manual, Univ. California, Berkeley, 2001.

[25] J. Csicsvari, H. Hirase, A. Czurko, and G. Buzsaki, "Reliability and state dependence of pyramidal cell-interneuron synapses in the hippocampus: An ensemble approach in the behaving rat," Neuron., vol. 21, no. 1, pp. 179-189, 1998.

[26] J. Csicsvari, H. Hirase, A. Czurko, A. Mamiya, and G. Buzsaki, "Oscillatory coupling of hippocampal pyramidal cells and interneurons in the behaving rat," J. Neurosci., vol. 19, no. 1, pp. 274-287, 1999.

[27] M. Dagtekin, W. Liu, and R. Bashirullah, "A multi channel chopper modulated neural recording system," Proc. IEEE Engineering in Medicine and Biology Meeting, pp. 757-760, 2001. 


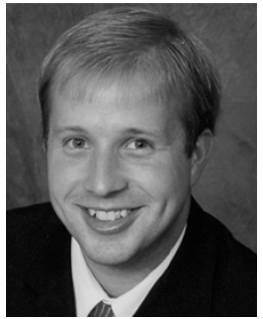

Roy H. Olsson, III (S'99-M'05) was born in Nevada, M.O., in 1976. He received the B.S. degrees (summa cum laude) in electrical engineering and in computer engineering from West Virginia University in 1999 and the M.S. and Ph.D. degrees in electrical engineering from the University of Michigan, Ann Arbor, in 2001 and 2004.

He interned at Lucent Technologies, where he designed mixed signal electronics for cellular phone applications. From 1999 to 2004, he was a Research Assistant in the Center for Wireless Integrated MicroSystems (WIMS) at the University of Michigan, where his doctoral research was in the development of implantable electronics for neural recording applications including amplification, multiplexing, and data compression circuitry. In July 2004, he joined the MEMS Device Technologies Group at Sandia National Laboratories, Albuquerque, NM as a Senior Member of Technical Staff. His current research areas include RF microresonators, RF transceivers, neural prosthetics, bio-interface electronics and low noise readout circuits for optical MEMS sensors.

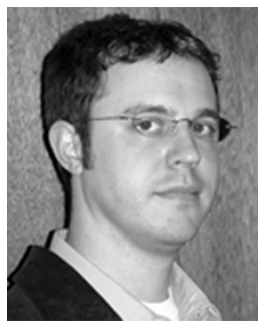

Derek L. Buhl received the B.A. degree in psychology from Rutgers University, Newark, , in 1999. He received the Ph.D. degree in behavioral and neural sciences at Rutgers in Newark, N.J. in November of 2004. He completed his thesis work with Dr. G. Buzsaki, investigating the generation of hippocampal network patterns, namely theta (5-9 $\mathrm{Hz})$, gamma (30-80 Hz), and fast-field "ripple" oscillations (140-200 Hz) and how these patterns participate in neuronal plasticity in genetically modified mice and developing rats. Interested in learning and utilizing new techniques and technologies, he collaborated with the laboratory of K. D. Wise, performing the in vivo beta testing of numerous silicon probes with advanced integrated circuits that were developed by R. H. Olsson, III. He will soon begin a post-doctoral fellowship at the EPFL in Lausanne, Switzerland.

Dr. Buhl is a member of the Society for Neuroscience and the American Physiological Society.

Anton M. Sirota received the M.A. degree (with honors) in physics and mathematics from Moscow Institute of Physics and Technology in 1999, and is currently working towards the Ph.D. degree in behavioral and neural sciences at Rutgers in Newark, NJ. His research focuses on experimental and theoretical analysis of the mechanism of information transfer between neocortex and hippocampus. Silicon probes developed by the Michigan group are the main experimental tool in his research.

Anton Sirota is a member of the Society for Neuroscience.

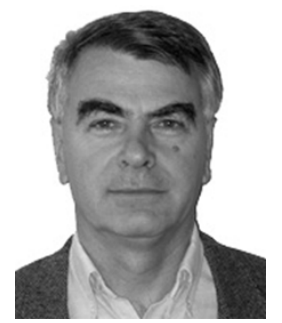

Gyorgy Buzsaki received the M.D. from the University of Pecs, Pecs, Hungary in 1974 and the Ph.D. degree in neuroscience from the Academy of Sciences, Budapest in 1984.

From 1975 to 1986, he was an Associate Professor with the Institute of Physiology at the University of Pecs School of Medicine. In 1984-1985 he was a visiting Associate Professor at the University of Lund, Lund, Sweden. He served as the J.D. French Foundation Fellow with the Department of Neurosciences at UC San Diego from 1986-1988 before becoming an Associate Professor in-Residence at UC San Diego in 1988. In 1990, he joined the faculty as a Professor at the Center for Molecular and Behavioral Neuroscience at Rutgers University, Newark, NJ. His current research interests include imaging local network activity in vivo, in vivo study of network cooperation in the hippocampus, and study of the hippocampal interneuronal network.

Dr. Buzsaki has served on the editorial board of Neuron; Neuroscience (Section Editor); Journal of Neuroscience (1994-2003); Journal of Neurophysiology; Behavioral Brain Research; Epilepsia; Hippocampus; Thalamus; Neuroscience Research Communications; Behavioral Neuroscience (1992-1996); Restorative Neurology and Neuroscience (1990-1996). He received the Excellence in Research Award from Rutgers University in 1995, the Distinguished Lecturer award from the Collége de France in 1998 and the Krieg Cortical Discoverer Award from the Cajal Club, American Association of Anatomists in 2001. Dr. Buzsaki became a Fogarty International Senior Fellow, Paris, France, in 2000, a Foreign member of the Hungarian Academy Sciences in 2001 and was elected Fellow of AAAS in 2004.

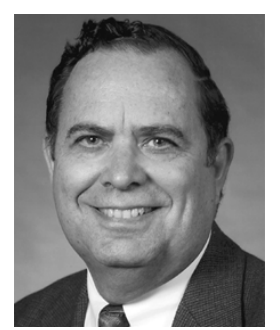

Kensall D. Wise (S'61-M'69-SM'83-F'86) received the B.S.E.E. degree with highest distinction from Purdue University in 1963 and the M.S. and $\mathrm{Ph} . \mathrm{D}$. degrees in electrical engineering from Stanford University in 1964 and 1969, respectively.

From 1963 to 1965 (on leave 1965-1969) and from 1972 to 1974 , he was a Member of Technical Staff at Bell Telephone Laboratories, where his work was concerned with the exploratory development of integrated electronics for use in telephone communications. From 1965 to 1972, he was a Research Assistant and then a Research Associate and Lecturer in the Department of Electrical Engineering at Stanford, working on the development of integrated circuit technology and its application to solid-state sensors. In 1974 he joined the Department of Electrical Engineering and Computer Science at the University of Michigan, Ann Arbor, where he is now the J. Reid and Polly Anderson Professor of Manufacturing Technology and Director of the NSF Engineering Research Center for Wireless Integrated MicroSystems. His present research interests focus on the development of integrated microsystems for health care, process control, and environmental monitoring.

Dr. Wise organized and served as the first chairman of the Technical Subcommittee on Solid-State Sensors of the IEEE Electron Devices Society (EDS). He was General Chairman of the 1984 IEEE Solid-State Sensor Conference, Technical Program Chairman of the IEEE International Conference on Solid-State Sensors and Actuators (1985), and IEEE-EDS National Lecturer (1986). He served as General Chairman of the 1997 IEEE International Conference on Solid-State Sensors and Actuators. He received the Paul Rappaport Award from the EDS (1990), a Distinguished Faculty Achievement Award from the University of Michigan (1995), the Columbus Prize from the Christopher Columbus Fellowship Foundation (1996), the SRC Aristotle Award (1997), and the 1999 IEEE Solid-State Circuits Field Award. In 2002 he was named the William Gould Dow Distinguished University Professor at the University of Michigan. $\mathrm{He}$ is a Fellow of the AIMBE and a member of the United States National Academy of Engineering. 